\title{
Integrated SDN/NFV Orchestration for the Dynamic Deployment of Mobile Virtual Backhaul Networks over a Multi-layer (Packet/Optical) Aggregation Infrastructure [Invited]
}

\author{
Ricardo Martínez, Arturo Mayoral, Ricard Vilalta, Ramon Casellas, Raül Muñoz, Stephan Pachnicke, \\ Thomas Szyrkowiec, and Achim Autenrieth
}

\begin{abstract}
Future 5G networks will bring important challenges to network operators such as the traffic load increase mainly due to the proliferation of mobile broadband communications. This will force Mobile Network Operators (MNOs) re-designing and investing on their infrastructures (e.g., new equipment for Radio Access Network - RAN -, backhaul, etc.) to cope with such data growth. Aiming at lowering both CapEx and OpEx, current networking trends on network virtualization, Software Defined Networking (SDN) and Network Function Virtualization (NFV) provide an appealing scenario to flexibly deal with MNO's increase of traffic without over-dimensioning the deployed network resources.

To this end, we rely on an implemented SDN/NFV orchestrator which automatically serves MNO capacity requests by computing and allocating virtual backhaul tenants. Such backhaul tenants are built over a common physical aggregation network, formed by heterogeneous technologies (e.g., packet and optical) that may be owned by different infrastructure providers. MNO's RAN traffic is transported towards mobile core network (i.e. Evolve Packet Core, EPC) where required backhaul resources are tailored to the capacity needs. The EPC functions are virtualized within the cloud (i.e., vEPC) leveraging the NFV advantages. This increases MNO flexibility where cloud resources are instantiated according to EPC needs. The goal of the SDN/NFV orchestrator is to jointly allocate both network and cloud resources deploying virtual backhaul tenants and vEPC instances for a number of MNOs with different service and capacity requirements. Each MNO's backhaul is isolated and controlled independently via a virtualized SDN controller (vSDN) deployed in the cloud.

The SDN/NFV orchestrator architecture is detailed and experimentally validated in a setup provided by CTTC and ADVA. Specifically, upon an MNO request the orchestrator instantiates the VEPC and VSDN functions in the cloud and then composes the MNO's backhaul tenant over a multi-layer (packet and optical) aggregation network.
\end{abstract}

Index Terms-Software Defined Networking, Network Function Virtualization, Virtual Mobile Backhaul Networks, Multi-Layer Networks.

\section{INTRODUCTION}

$\mathbf{F}$ uture $5 G$ networks will considerably challenge network operators aiming at dealing with stringent network requirements: Such requirements are imposed by new expected advanced services (e.g., real time high quality services, assisted driving, smart metering, etc.). Specifically, these

Manuscript received June 15, 2016.

R. Martínez, R. Casellas, R. Vilalta and R. Muñoz are with CTTC, Spain (e-mail: ricardo.martinez@cttc.es).

S. Pachnicke is with Kiel University (CAU), Kiel, Germany.

T. Szyrkowiec and A. Autenrieth are with ADVA Optical Networking SE, Martinsried, Germany. include not only a substantial increase of the overall data rate (x100 of $4 \mathrm{G}$ peak rates), but also reduced end-to-end latency (10 ms or less), enhanced energy-efficiency, massive connectivity (e.g., IoT), guaranteed QoS, etc. [1]. The goal, for network operators, is to design and develop 5G network architectures from an end-to-end perspective (i.e., access, metro and core network segments) in a cost-efficient manner.

Mobile broadband communications are the drivers increasing the traffic volume in 5G networks. Hence, mobile network operators (MNOs) will need to invest in new solutions and/or enhancing their infrastructure: Radio Access Technology (RAT), Fronthaul, Backhaul, etc. Typically, as traffic increases, MNOs deploy new dedicated network appliances for both control and data plane. These deployments are generally over-dimensioned considering the load foreseen for the next three to five years peak hours [2]. By doing so, some network resources may be (frequently) wasted resulting in a not effective strategy in terms of CapEx and OpEx. A more flexible, agile and cost-efficient solution to attain a better use of available resources relies on leveraging the features provided by Network Function Virtualization (NFV) and Software-Defined Networking (SDN) [3].

NFV deploys network functions, typically allocated in dedicated hardware, as software instances referred to as virtual network functions (VNFs) [4]. VNFs are executed in commercial off-the-shelf (COTS) hardware (servers) within data centers (DCs) enabled by virtualization techniques. This provides important benefits to network operators in terms of cost, flexibility (reduced time to market, avoiding vendor lock-in, enhanced scalability, etc.), openness, homogeneity of configuration, migration, etc. NFV is applicable to any data plane packet processing and control plane function in fixed and mobile network infrastructures [5] [6]. In the MNO context, NFV is addressing the use case for virtualizing the mobile Core (vEPC) which in the last years has received significant attention [6] [7].

On the other hand, SDN deals with a logically centralized control enabling the network programmability by decoupling data and control planes [8]. SDN offers a logical plane abstraction, hiding vendor-specific hardware which fosters multi-vendor interoperability. Such an abstraction enables network virtualization, that is the partitioning (slicing) of the physical infrastructure to create multiple co-existing and independent network tenants on top of it [3].

In light of the above benefits and to deal with the expected 5G MNO's capacity increase, virtualization of both network functions (via NFV) and infrastructure (via SDN) can be efficiently exploited. This work assumes that a number of MNOs 
owning their radio access network (RAN) equipment (i.e., eNodeBs) are connected to a common multi-layer (packet and optical switching) aggregation infrastructure. This network, that may be owned by different physical infrastructure providers, is then shared by MNOs to deploy their backhaul and avoids deploying new dedicated network elements. The physical aggregation network is partitioned (via SDN) to compose MNO's virtual backhaul tenants on top of it. MNO's backhaul tenant resources are computed to be adjusted to the actual MNO traffic demands. The MNO's core EPC functions (i.e., Mobile Management Entity - MME, Serving Gateway - SGw, Packet Data Network Gateway, - PGw, etc.) [9] are virtualized into the DCs which are reached via the shared multi-layer aggregation network.

This paper, based on the work produced by authors in [10], presents and experimentally validates an integrated SDN/NFV orchestration architecture to dynamically compute and automatically deploy individual MNO virtual backhaul tenants with their corresponding vEPC instantiated into the DC. The orchestrator system automatically coordinates the virtualization of heterogenous transport technologies (packet and optical) as well as computing and allocating the cloud resources for deploying VNFs such as vEPC and vSDN controller [3] (see Fig. 1). A proof-of-concept is carried out in a joint setup formed by: the CTTC SDN/NFV orchestrator, CTTC packet and DC domains, and the ADVA Optical Network Hypervisor (ONH) [11] used for controlling the optical domain between packet networks.

The rest of this paper is organized as follows: in Section II, we overview related work for virtualization of both network infrastructure and functions. In Section III, the adopted network scenario is detailed by means of an example where MNO's SDN-controlled backhaul tenants are deployed over a common physical aggregation infrastructure. Section IV describes the implemented SDN/NFV orchestration architecture. The experimental proof-of-concept over a CTTC-ADVA setup is reported in Section V. Finally, we conclude the paper in Section VI.

\section{RELATED WORK}

In the case of network virtualization Virtual Network Providers aggregate infrastructures from different Physical Infrastructure Providers enabling that Virtual Network Operators can offer their services over a common and shared infrastructure. In the last years a number of works has been produced virtualizing network infrastructures composed by a single (e.g., optical domain [11]) or multi- technologies (packet and optical domains e.g., [12], wireless and optical technologies, e.g., [13], etc.). Regardless of the underlying network technologies, a key element for deploying (virtual) network tenants over a common infrastructure is the use of a logically centralized control entity. This entity is responsible for providing the network slicing. That is, network resources are partitioned and abstracted and used to compose network tenants. The computation and creation of the network tenants may satisfy specific services' demands in terms of endto-end connectivity, latency, bandwidth, availability, etc. The adoption of a centralized SDN controller actually provides the required abstraction function of the physical network infrastructure being controlled.

For the network virtualization, two abstraction models/strategies can be used [11]: single virtual node and $a b$ stract link model. The former represents either a completer or a partial network topology as a single virtual node. By doing so, the internal domain topology is hidden by the abstraction function. Ports / interfaces of the virtual node are in general simply mapped to the real physical interfaces of the complete / partial network domain. On the other hand, the abstract link model provides a summarized view of the internal topology of the network domain. For instance, the abstract view on this model may have a representation of inter-connected domain border nodes with their external links. Herein we will concentrate on the single virtual node approach for abstracting network domains. In the context of multiple domains managed by different physical infrastructure providers, the virtual node approach may be preferred since internal domain details do not need to be shared.

NFV targets specific network functions being virtualized in commodity hardware (COTS). As said, this brings cost reductions, accelerates network and service deployment, etc. Those advantages of NFV are combined with the virtualization of physical network elements (e.g., packet and optical switches). Hence, both network and IT resources are jointly considered when composing multi-tenants [6], [12][14] (for fixed and mobile networks and services). NFV is applicable to all network segments (i.e., access, metro and core) covering a number of network functions such as virtual Border Network Gateway (vBNG) [5], virtual mobile core (vEPC) [2] [6] [7], etc. The virtualization of specific network functions (VNFs) can be sorted by two models partial or full [2]. In the partial model only selected network functions (e.g., control plane-oriented as EPC MME) are virtualized in the DC whereas other functionalities (e.g., EPC SFGw/PGw data plane's forwarding and tunneling) are kept in dedicated network elements. Conversely, in the full model all (control and data plane) functional entities are implemented as VNFs running in DC's virtual machines (VMs).

The control and management of each individual network tenant is handled by a centralized SDN controller. In other words, each network tenant has its own (isolated) network infrastructure and functions and the control and configuration is attained by an independent SDN controller. The dynamic deployment of SDN controllers per tenant also leverages the NFV capabilities. That is, DC's cloud resources are used to instantiate a virtual SDN (vSDN) controller for each network tenant [3], [15].

For the dynamic deployment of MNOs' virtual backhaul infrastructures along with VNFs for vEPC and vSDN, herein we rely on some of the aforementioned concepts and solutions.

\section{DEPloyment OF SDN-CONTROLlED MNO ViRTUAL BACKHAUL}

Figure 2 depicts the physical topology of the considered multi-layer (packet and optical switching) aggregation infrastructure. This network allows the connectivity between the MNO's RAN to the DC domains where specific VNFs for vEPC and vSDN are instantiated. We assume that all the mobile core functions are virtualized. Thus, herein we rely on the full virtualization model of the EPC [2] and we extend it with the virtualization of backhaul network infrastructure to connect the RAN and the vEPC domains.

The aggregation network leverages the statistical multiplexing provided by packet switching (MPLS) and the huge transport capacity of optical switching applying multi-layer 


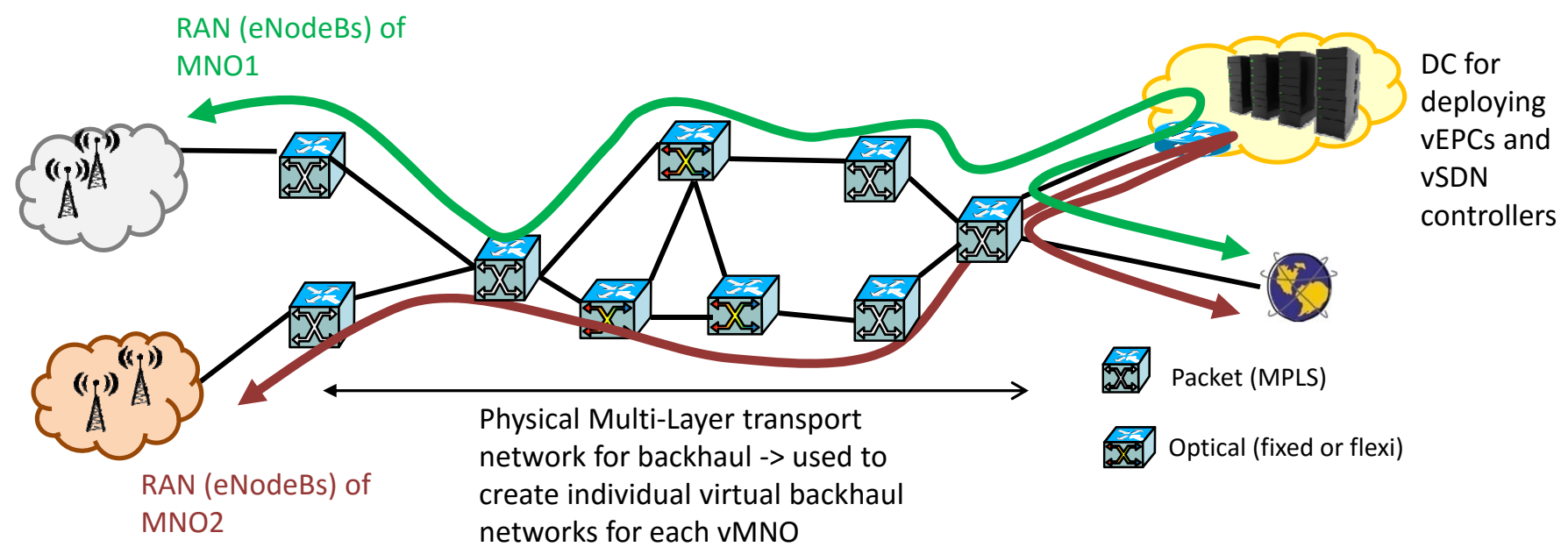

Fig. 1. Support of multiple MNO's virtual backhaul infrastructure over a common multi-layer aggregation network

grooming techniques [16]. An MNO creating/modifying its backhaul capacity is built over the aggregation network as interconnected virtual packet domains. The MNO SDN controller's vision (deployed as a VNF) is an abstraction of a set of connected packet domains (via an optical connection) providing the connectivity between the RAN and vEPC. Each abstracted packet domain is represented by a single (virtual) packet switch node. The interfaces of such a node are mapped to the physical incoming / outgoing links of a packet flow. In the example (Fig. 2), for the MNO1 topology the virtual packet node of the domain linked to the RAN is formed by ingress link $\mathrm{A}$ and egress link $\mathrm{E}$ of the corresponding physical packet network.

The view of each MNO's vSDN controller allows then to dynamically compute and set up packet (MPLS) tunnels for backhauling upcoming mobile (Long Term Evolution, LTE) control and user traffic flows (i.e., S1-MME and S1$\mathrm{U}$ interfaces [9]) between the RAN and vEPC functions. To this end, the vSDN controller uses the OpenFlow protocol (OFP) to configure each virtual packet domain. A hypervisor layer exists between the vSDN controller and the network infrastructure (Section IV) performing abstraction, isolation and translation functionalities mapping vSDN control messages to their physical resources. The connectivity within the DC network domain is virtualized connecting the core packet domain and the deployed cloud VNFs (i.e., vSDN controller and vEPC).

In the example, each virtual MNO's backhaul infrastructure is created connecting (at the packet level), via a direct link/tunnel with the required capacity, the two abstracted packet nodes linked to both the RAN and the mobile core domains. The resulting virtual backhaul topology mostly depends on the available physical network as well as the virtual network strategy (i.e., partitioning and composition) being adopted. That said, it is worth mentioning that the targeted automatic MNO backhaul creation supports the deployment of more advanced network topologies (e.g., mesh, rings). Indeed, these topologies are needed, for instance, by an MNO owning a number of remote RAN domains. The traffic generated by each RAN domain can be aggregated into higher capacity links and steered towards the EPC. Additionally, more complex virtual backhaul networks also provide appealing capabilities that $5 \mathrm{G}$ services would require such as effective recovery mechanisms (e.g., disjoint paths), QoS differentiation, etc.

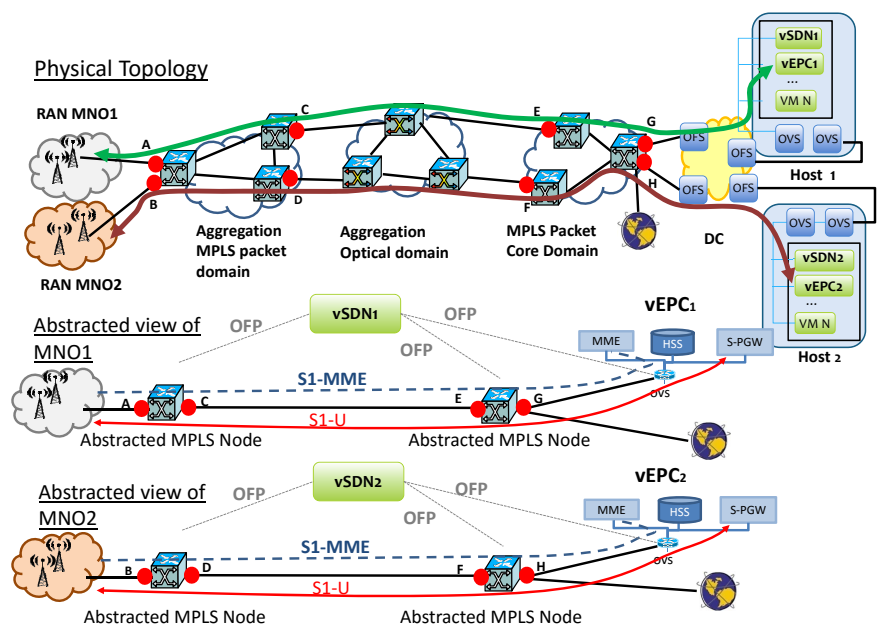

Fig. 2. Physical multi-layer aggregation network connecting RANs and DCs and abstracted view of the backhaul networks per MNO

\section{SDN/NFV ORChESTRATION ARCHITECTURE FOR}

DyNAMIC DEPLOYMENT OF MNO ViRTUAL BACKHAUL

The SDN/NFV orchestrator architecture used to deploy MNO's virtual backhaul tenants with the respective DC's VNFs is represented in Fig. 3. The NFV orchestrator deploys VNFs on the compute domain within the DC/cloud. This infrastructure along with the network domain (formed by the packet and optical switches) constitute the so-called NFV infrastructure (NFVI) [4]. Whenever a new VNF (e.g., vSDN controller or vEPC) needs to be deployed, the VNF Manager is created to deploy and handle the targeted VNFs.

In Fig. 3, following a bottom-up description, besides the network infrastructure (packet and optical switches of the aggregation and DC domains), individual control plane instances are dedicated to configure the network elements forming an individual domain. These controllers / control plane elements are part of the Virtual Infrastructure Manager (VIM) defined by the ETSI NFV architecture [4]. Four independent controllers are deployed: i) an SDN controller 
for the (MPLS) packet domain connected to the RAN; ii) an ONH used for configuring the optical network domain; iii) an SDN controller for the packet network connected to the DC; iv) an SDN controller handling the flows within the DC networking.

The Multi-Domain SDN Orchestrator (MSO) is a unified transport network operating system which takes over of the composition of end-to-end provisioning services across multiple domains of the aggregation network at an abstraction level (i.e., using virtual node representation). Specifically, the MSO is a controller of controllers whose implementation follows the IETF Application-Based Network Operations (ABNO) architecture [17]. The MSO coordinates, in a hierarchical way, the underlying controllers to configure/program the computed physical network resources on the involved (traversed) domains for setting up a new/modified end-to-end service. This model allows the SDN controllers abstracting their respective domain topology and resource information towards the MSO. Such a domain abstraction, in turn, allows ensuring the scalability of the whole control system.

Another relevant architectural element is the MultiDomain Network Hypervisor (MNH) [18]. The MNH partitions and aggregates the physical resources (i.e., nodes, links, optical spectrum, etc.) in each domain into virtual resources. Next, virtual resources are interconnected to compose the MNO's backhaul tenants. Therefore the MNH is responsible for the abstraction of the backhaul tenant at the packet layer. Recall that such backhaul tenants are controlled by instantiated vSDN controllers. That is, the MNH provides the topology vision to the vSDN controller about each MNO's backhaul infrastructure. The MNH provides the hypervisor functions such as abstraction, translation and isolation (of the backhaul tenants) to each vSDN controller as well as triggers the creation, modification and deletion of the virtual backhaul infrastructure in response to received MNO demands.

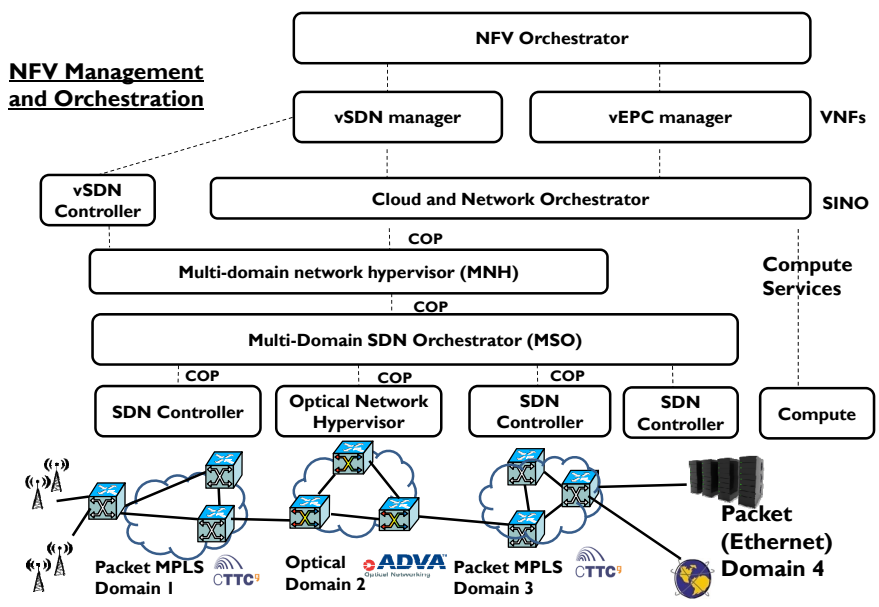

Fig. 3. SDN/NFV orchestration architecture provising MNO backhaul virtual networks

The Cloud and Network Orchestrator handles the coordination and management of cloud resources (VMs) and network resources within the aggregation infrastructure. It provides an ecosystem for a cloud and network operating system towards deploying jointly MNO virtual backhaul and its vEPC functions. It relies on a southbound interface which enables the communication with the $\mathrm{MNH}$ via the so- called Control Orchestration Protocol (COP) [19]. The COP API is a transport application interface used to basically retrieve (abstracted) network topology, to serve connectivity requests and to perform end-to-end path computation. For the cloud resources, the Cloud and Network Orchestrator has a compute controller used for configuring the compute domain (i.e., VNF instantiation) and an hypervisor control used to configure the VMs over the physical compute domain. The Cloud and Network orchestrator provides the functionalities being defined as the VIM entity in the ETSI NFV architecture. The Cloud and Network Orchestrator in this work is also referred to as SDN Integrated IT and Network Orchestrator (SINO).

Finally the NFV Orchestrator manages the physical and virtual resources to support the applications requesting the creation / enhancement of the MNO virtual backhaul as well as the corresponding VNFs (vEPC and vSDN) in the cloud.

\section{Experimental Proof of Concept}

This section addresses the experimental proof-of-concept (as a validation) of the implemented SDN/NFV orchestrator functionalities. Upon MNO demands, dynamic and automatic computation and deployment of a packet backhaul tenant over the aggregation network is performed along with instantiating VNFs (i.e., vEPC and vSDN). The proof-ofconcept has been jointly conducted by CTTC and ADVA. In the following, the workflow is detailed, which is used by the $\mathrm{SDN} / \mathrm{NFV}$ orchestrator to coordinate the required functions and control of data plane elements described in Section IV. It is worth saying that if the workflow is completed, the targeted backhaul tenant for the MNO request is successfully deployed. Otherwise, if no sufficient (networking or cloud) resources are available to serve the MNO request, this is blocked by the SDN/NFV orchestrator. The experimental setup (including the network elements, controllers and DC infrastructure) used for validating the workflow is described showing main protocol analyzer captures demonstrating the exchanged messages within the SDN/NFV orchestrator.

\section{A. Description of the WorkFlow}

Figure 4 shows the workflow among the involved functional blocks of the SDN/NFV orchestrator (SINO) to manage the creation of an SDN-controlled virtual backhaul and the corresponding vEPC. The whole process is divided into two macroscopic steps:

- Step 1: the NFV orchestrator requests the creation of the vSDN controller (to control via OFP the virtual backhaul) and the vEPC mobile core (control and user plane) functions within the DC. Specifically, those requests are delegated to the respective VNF managers. The VNF managers in turn communicate with the DC's Compute Control requiring the creation of VMs (specifying cloud resources in terms of CPU and memory) with the respective operating system image for the VNF implementations (i.e., vEPC and vSDN). The response determines the IP and MAC addresses of the involved elements and functions: vSDN and vEPC (including PGw, SGw and MME).

- Step 2: the creation of the MNO virtual backhaul is carried out. This process entails building the virtual backhaul and enabling the connectivity to the corresponding created vSDN (in Step 1). To do that, the 
$\mathrm{MNH}$ receives and processes the request (including the IP address of the vSDN). The MNH computes, using the abstract view of the underlying transport network (at the packet level), the domain sequence connecting the MNO's RAN and the vEPC within the DC. To this end, the service requirements to be offered by the MNO such as peak data rate or maximum tolerated end-to-end latency are taken into account. In the considered multilayer aggregation network, it is needed first that the traversed packet domains are interconnected via an optical connection triggered by the MSO. In other words, MNH computes a sequenced set of virtual packet nodes which in the physical infrastructure are connected via an optical domain. The configuration of the multi-layer physical infrastructure is handled by the MSO. When the optical connection is set up (using the ADVA ONH), at the packet level, all the domains are interconnected. For those packet domains the MSO subsequently requests packet flow provisioning specifying the ingress/egress links of those domains to derive the abstracted (virtual) packet node forming the targeted virtual backhaul. It is worth mentioning that this process is performed twice to support bidirectional packet communications within the backhaul. Last but not least, a Layer 2 flow within the DC infrastructure (Ethernet) is created to connect the virtual packet node with the vEPC. Once the virtual backhaul connectivity is ready, this is notified to the NFV orchestrator, and at that time, the vSDN has a view of the virtual packet backhaul used to transport both LTE control and user plane traffic between the RAN and the deployed vEPC.

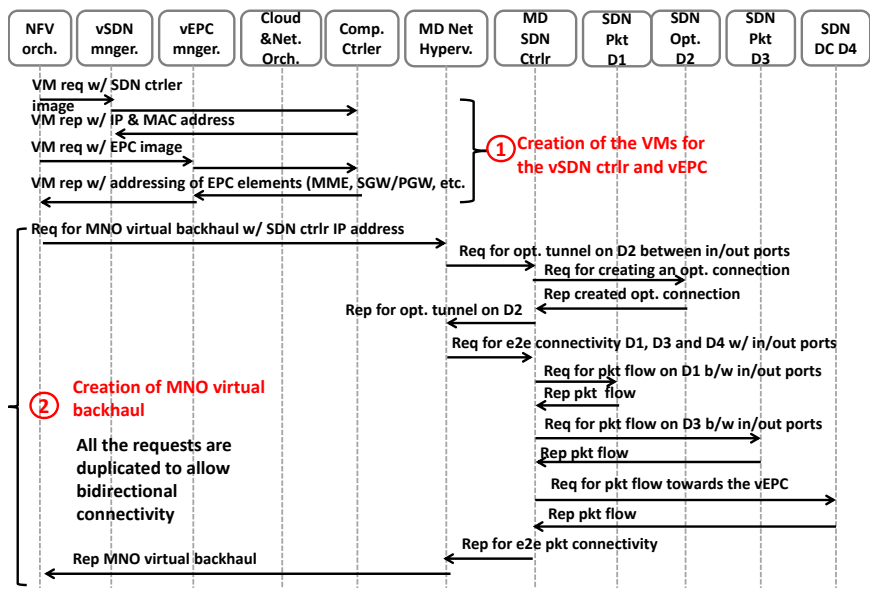

Fig. 4. Workflow for provisioning MNO virtual backhaul network and VNFs

\section{B. Experimental Setup and Validation}

Figure 5 depicts the interconnection between the CTTC SDN/NFV orchestrator located at Barcelona (Spain) and the ADVA ONH in Meiningen (Germany). For the sake of clarification, the setup is conducted at the control plane level. No data plane configuration is finally realized once the whole $\mathrm{SDN} / \mathrm{NFV}$ orchestration process is completed. To enable the remote communication within the $\mathrm{SDN} / \mathrm{NFV}$ orchestrator (SINO), in particular, the MSO located at CTTC and the ONH placed at ADVA, an OpenVPN tunnel between CTTC and ADVA has been established over the public Internet. As a consequence, the MSO via the COP protocol sends (routed over the OpenVPN tunnel) optical connection requests that will allows the connectivity between packet domains connected to both RAN and DC. The rest of the SDN controllers are provided by CTTC and configure the packet domains connected to the RAN, the core network and DC.

The vEPC implementation is based on the CTTC LENA emulator (NS-3 simulator) [20]. In a single VM equipped with two network adapters (one for the S1 interface and another for the SGi interface) needed control and user plane network functions for the creation/tunneling of mobile connections (bearers) are deployed: the EPC MME and the $\mathrm{SGw} / \mathrm{PGw}$ along with the required interfaces between them (i.e., S11). The SGw and $\mathrm{PGw}$ are implemented within the same software process. In the vEPC implementation, no authentication functionality (e.g., HSS) is implemented.

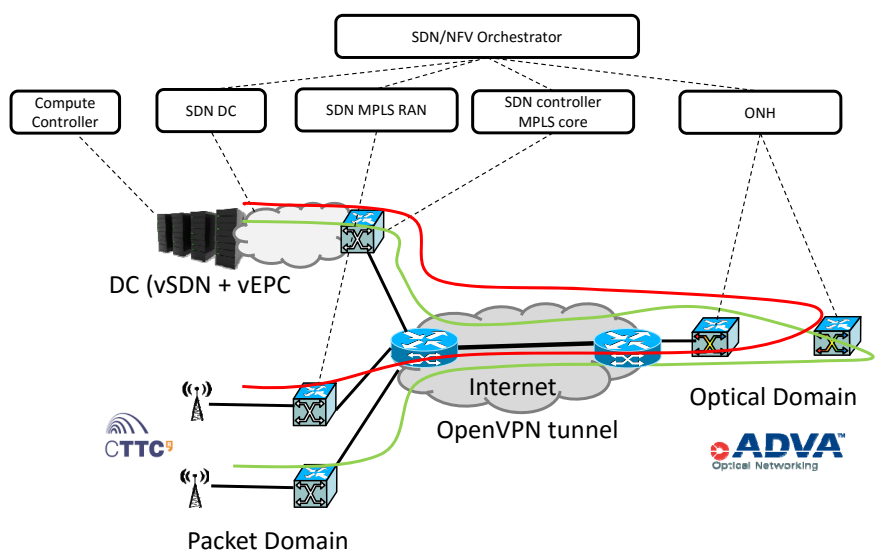

Fig. 5. Topology of the connectivity between CTTC SDN/NFV Orchestrator and the ADVA ONH

The experimental validation to create MNO's VNFs and the backhaul tenant using the SINO architecture is shown in the captured messages in Fig. 6. Following the workflow sequence, first a client (CLIENT in Fig. 6) MNO requests to the SINO the allocation of two VMs. These VMs host the targeted vEPC and vSDN controllers for the MNO demand. In the setup both the SINO and the MNH are deployed in the same building block (SINO-MNH) in Fig. 6. The used commands to instantiate VMs are implemented in a RESTful API (POST / create_vm/ method). As mentioned, two separated VM requests are made to accommodate the needed VNFs. As shown in the figure, the required time (requestresponse) to create the VMs and instantiate the vSDN controller and vEPC functions takes around 22s.

Once VMs are deployed, by means of another RESTful API (POST /virtual_network/ method) the client MNO triggers the request to create the virtual backhaul network. This request is processed, as detailed in the workflow, by the network hypervisor (i.e., SINO-MNH) and served by the multi-domain SDN orchestrator (i.e., MSO). The message sent by SINO-MNH to the MSO is made using the RESTful POST/restconf/config/calls/call/ method. In this message the IP addressing of both the vEPC core network functions and the vSDN within the DC are passed as contained information. The MSO is responsible for coordinating among the different packet and optical domains, the end-to-end connectivity between both the MNO RAN and the vEPC and the vSDN controller 
with the SINO-MNH. To provide the connectivity at the packet layer though the multi-layer aggregation network, the MSO requests the establishment of an optical tunnel within the optical domain to the ADVA MNH (simply labeled $A D V A$ in the following). Once the optical tunnel is created, at the packet level the domains adjacent to both the RAN and the DC networks are connected. Next, it is necessary to create the packet flows from the MNO's RAN and the deployed vEPC. To do that, the MSO entity communicates with the packet domains' controllers (SDN-CTL-1 and SDN-CTL-2). The RESTful API messages to establish the packet flows requested by the MSO are: PUT /restconf/config/oepndaylight-inventory/ and PUT / controller/nb/v2/flowprogrammer/ methods.

The request and received response for the optical tunnel establishment connecting the packet domains takes around $10 \mathrm{~ms}$ (in Fig. 6, it is reflected by the exchange of messages between MSO and ADVA ONH). It is worth mentioning that in this proof-of-concept we assumed that the optical tunnel was pre-established (i.e., no transceiver configuration was done). This is why ADVA optical hypervisor response is relatively fast. Conversely, if the optical transceivers need to be tuned and configured, the overall establishment of the optical tunnel would take hundreds of milliseconds.

Once the optical tunnel is set up, it becomes a (virtual) packet link connecting both packet domains. Then, the MSO communicates with each computed packet domain SDN controller to request the required packet flow configuration. In general, this takes around $11 \mathrm{~ms}$. The complete set up of the virtual backhaul tenant is done in $78 \mathrm{~ms}$. This includes not only the request/response messages elapsed time for setting up the optical tunnel and the packet domain configuration, but also processing time (i.e., path computation, topology updating, etc.) performed within the MSO.

\section{Conclusions}

In future $5 \mathrm{G}$ networks, mobile broadband communications will dramatically increase the data traffic. To cope with this, traditionally MNOs invest with new appliances on their infrastructures (i.e., RAN, backhaul/fronthaul networks, etc.) following over-dimensioned strategies. This results in a non cost-effective solution since some of the rolled out network resources may not be fully utilized. Herein we seek for a more flexible, agile and cost-efficient solution to better tailor the MNO capacity needs with the deployed network resources (in the backhaul). To that end, we exploit the benefits provided by both SDN and NFV concepts. Basically we implement an SDN/NFV orchestrator which according to the MNO capacity demands automatically computes and allocates backhaul network tenants on top of a common physical aggregation network. The creation of the (virtual) backhaul provides the connectivity between the MNO's RAN domains and the mobile core functions (EPC). For the latter, the EPC is virtualized in COTS hardware (vEPC) within the DC. The created MNO's backhaul tenant is completely isolated and controlled by its own SDN controller which is also dynamically deployed as a VNF (vSDN) within the DC. The SDN/NFV orchestrator architecture has been detailed along with the required workflow used to deploy an MNO's backhaul network and respective vEPC and vSDN from the scratch. Finally, a proof-of-concept has been experimentally validated such as a global architecture (functional entities and interfaces) over a multi-layer aggregation network provided by the CTTC SDN/NFV orchestrator and the ADVA $\mathrm{ONH}$.

\section{ACKNOWLEDGMENTS}

This work was partially funded by the EC's FP7 IP COMBO project under grant agreement 317762 and by the Spanish MINECO DESTELLO project (TEC2015-69256-R).

\section{REFERENCES}

[1] NGMN Alliance, "5G White Paper," February 2015.

[2] T. Taleb, et. al., "EASE: EPC as a Service to Ease Mobile Core Deployment over Cloud," IEEE Network, vol. 29, no. 2, March/April 2015.

[3] R. Muñoz, et. al., "Integrated SDN/NFV Management and Orchestration Architecture for Dynamic Deployment of Virtual SDN Instances for Virtual Tenant Networks," IEEE/OSA J. Opt. Commun. Netw. (JOCN), vol. 7, no. 11, November 2015.

[4] ETSI White Paper No. 3, "Network Function Virtualisation (NFV)," October 2014.

[5] C. Lange, D. Kosiankowski, and A. Gladisch, "Use-Case Based Cost and Energy Efficiency Analysis of Virtualization Concepts in Operator Networks," in in Proc. of European Conference on Optical Communications (ECOC), Valencia, Spain, September 2015.

[6] M. R. Sama, et. al., "Software-Defined Control of the Virtualized Mobile Packet Core," IEEE Commun. Mag., vol. 53, no. 2, February 2015.

[7] H. Hawilo, A. Shami, M. Mirahmadi, and R. Asal, "NFV: State of the Art, Challenges, and Implementation in Next Generation Mobile Networks (vEPC)," IEEE Network, vol. 28, no. 2, November/December 2014.

[8] Open Networking Foundation (ONF), "SDN Architecture Overview (version 1.1) - ONF TR-504," November 2014.

[9] 3GPP, "E-UTRA and E-UTRAN; Overall description - TS 36600," March 2012.

[10] R. Martinez, et. al., "Integrated SDN/NFV Orchestration for the Dynamic Deployment of Mobile Virtual Backhaul Networks over a Multi-Layer (Packet/Optical) Aggregation Infrastructure," in in Proc. of Optical Fiber Communication Conf. and Expo. (OFC), Anaheim, California, March 2016.

[11] A. Autenrieth, et. al., "Evaluation of Virtualization Models for Optical Connectivity Service Providers," in in Proc. of Optical Network Design and Modeling (ONDM), Stockholm, Sweden, May 2014.

[12] R. Vilalta, A. Mayoral, R. Munoz, R. Casellas, and R. Martinez, "Multitenant Transport Networks with SDN/NFV," IEEE / OSA J. of Lightwave Technology, vol. 34, no. 6, March 2016.

[13] A. Tzanakaki, et. al., "Virtualization of Heterogeneous Wireless-Optical Network and IT Infrastructures in Support of Cloud and Mobile Cloud Services," IEEE Commun. Mag. vol. 51, no. 8, August 2013 .

[14] R. Muñoz, R. Casellas, R. Vilalta, and R. Martinez, "Dynamic and Adaptive Control Plane Solutions for Flexi-Grid Optical Networks Based on Stateful PCE," IEEE J. Lightw. Technol., vol. 32, no. 16, August 2014.

[15] A. Blenk, A. Basta, and W. Kellerer, "HyperFlex: An SDN Virtualization Architecture with Flexible Hypervisor Function Allocation," in in Proc. of IFIP/IEEE International Symposium on Integrated Network Management (IM2015), Ottawa, Canada, May 2015.

[16] R. Martinez, R. Casellas, and R. Munoz, "Experimental Validation / Evaluation of a GMPLS Unified Control Plane in MultiLayer (MPLS-TP/WSON) Networks," in in Proc. of Optical Fiber Communication Conf. and Expo. (OFC), Los Angeles, California, March 2012.

[17] D. King and A. Farre, "A PCE-Based Architecture for Application-Based Network Operations," IETF RFC 7491, March 2015.

[18] R. Vilalta, et. al., "Multidomain Network Hypervisor for Abstraction and Control of OpenFlow-enabled Multitenant Multitechnology Transport Networks," IEEE/OSA J. Opt. Commun. Netw. (JOCN), vol. 7, no. 11, November 2015. 


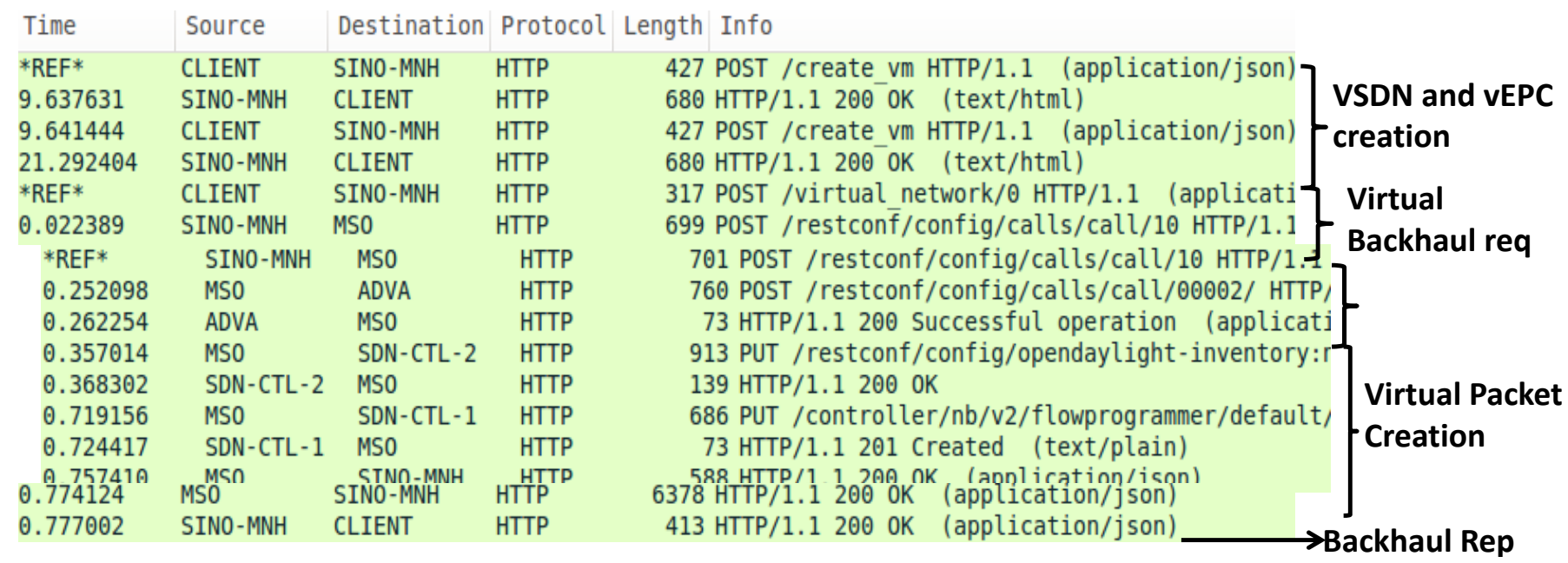

Fig. 6. Capture of the experimental control messages for setting up the VNFs and virtual backhaul network

[19] — - "The Need for a Control Orchestration Protocol in research Project on Optical Networking," in in Proc. of European Conference on Networks and Communications (EuCnC 2015), Paris, France, June 2015.

[20] CTTC LENA EPC/LTE Network Simulator, "http://networks.cttc.es/mobile-networks/software-tools/lena/."

Ricardo Martínez (SM'14) graduated and PhD in telecommunications engineering by the UPC-BarcelonaTech university in 2002 and 2007, respectively. He has been actively involved in several public-funded (national and EU) R\&D as well as industrial technology transfer projects. Since 2013, he is Senior Researcher of the Communication Networks Division (CND) at CTTC. His research interests include control and network management architectures, protocols and traffic engineering mechanisms for next-generation packet and optical transport networks within aggregation/metro and core segments.

Arturo Mayoral received the Graduate degree in telecommunications engineering from the Universidad Autonoma de Madrid, Madrid, Spain, in 2013, and is currently working toward the M.Sc. degree at the Universitat Politecnica de Catalunya, Barcelona, Spain. During 2012, he was an Intern Researcher in Telefonica I+D. In 2013, he started to work as a SW Developer and Research Assistant in CTTC within the ICT STRAUSS European-Japan Project.

Ricard Vilalta graduated in telecommunications engineering in 2007 and received a Ph.D. degree in telecommunications in 2013 , both from the Universitat Politècnica de Catalunya (UPC), Spain. $\mathrm{He}$ also has studied Audiovisual Communication at UOC (Open University of Catalonia) and is a master degree on Technology-based business innovation and administration at Barcelona University (UB). Since 2010, Ricard Vilalta is a researcher at CTTC, in the Optical Networks and Systems Department. His research is focussed on Optical Network Virtualization and Optical Openflow. He is currently a Research Associate at Open Networking Foundation.
Ramon Casellas (SM'12) graduated in telecommunications engineering in 1999 by both the UPC-BarcelonaTech university and ENST Telecom Paristech, within an Erasmus/Socrates double degree program. After working as an undergraduate researcher at both France Telecom R\&D and British Telecom Labs, he completed a Ph.D. degree in 2002. He worked as an associate professor at the networks and computer science department of the ENST (Paris) and joined the CTTC Optical Networking Area in 2006, with a Torres Quevedo research grant.

He currently is a senior research associate and the coordinator of the ADRENALINE testbed. He has been involved in several international R\&D and technology transfer projects. His research interests include network control and management, the GMPLS/PCE architecture and protocols, software defined networking and traffic engineering mechanisms. He contributes to IETF standardization within the CCAMP and PCE working groups. He is a member of the IEEE Communications Society and a member of the Internet Society.

Raül Muñoz (SM'12) graduated in telecommunications engineering in 2001 and received a Ph.D. degree in telecommunications in 2005, both from the Universitat Politècnica de Catalunya (UPC), Spain. After working as undergraduate researcher at Telecom Italia Lab (Turin, Italy) in 2000, and as assistant professor at the UPC in 2001, he joined the Centre Tecnològic de Telecomunicacions de Catalunya (CTTC) in 2002.

Currently, he is Senior Researcher, Head of the Optical Network and System Department, and Manager of the Communication Networks Division. Since 2000, he has participated in several R\&D projects funded by the European Commission's Framework Programmes (FP7 FP6 and FP5) and the Spanish Ministries, as well as technology transfer projects. He has led several Spanish research projects, and currently leads the European Consortium of the EUJapan project STRAUSS. His research interests include control and management architectures, protocols and traffic engineering algorithms for future optical transport networks.

Stephan Pachnicke (SM'12) received the M.Sc. degree in information engineering from City University, London, UK, in 2001 and the Dipl.-Ing. and Dr.-Ing. degrees in electrical engineering from TU Dortmund, Germany, in 2002 and 2005, respectively. In 2005 he also got the Dipl.-Wirt.-Ing. degree in business administration from Fern Universität, Hagen, Germany. In January 2012 he finished his habilitation on optical transmission networks. 
From 2007 until 2011 he was working as Oberingenieur at the Chair for High Frequency Technology, TU Dortmund. Afterwards he was with ADVA Optical Networking SE in the Advanced Technology Group (CTO Office), where he was leading (EU-funded) research projects on next generation optical access and fixed-mobile convergence. Since 2016 he has been Full Professor of Optical Communications at Christian-Albrechts-University of Kiel, Germany. He is author or co-author of more than 100 scientific publications and several (pending) patents. He is a member of VDE/ITG and a IEEE senior member.

Thomas Szyrkowiec TBC.

Achim Autenrieth TBC. 